\title{
Hardware Security of Emerging Non-Volatile Memory Devices under Imaging Attacks
}

\author{
Nhu Huynh \\ Department of Electrical and Computer \\ Engineering \\ The University of Texas at San Antonio \\ San Antonio, U.S.A \\ nhu.huynh@my.utsa.edu
}

\author{
Hebin Cherian \\ Department of Electrical and Computer \\ Engineering \\ The University of Texas at San Antonio \\ San Antonio, U.S.A \\ hebin.cherian@my.utsa.edu
}

\author{
Ethan C. Ahn \\ Department of Electrical and Computer \\ Engineering \\ The University of Texas at San Antonio \\ San Antonio, U.S.A \\ ethan.ahn@utsa.edu
}

\begin{abstract}
The emerging non-volatile memory (NVM) devices are currently changing the landscape of computing hardware. However, their hardware security remains relatively unexplored in the field. This is a critical research problem because given that they are non-volatile, sensitive information may be vulnerable to various physical attacks unless properly encrypted. In this work, we investigated security vulnerability of two emerging non-volatile memory devices (STT-MRAM and RRAM) against the most commonly available, non-destructive physical attack Scanning Electron Microscope (SEM) imaging. The central premise is that if any difference of memory cells in high resistance and low resistance (bit ' 1 ' and ' 0 ') states can be detected in SEM, stored data could possibly leak or be stolen by adversaries. It is concluded that unless advanced elemental analysis techniques such as energy dispersive $x$-ray spectroscopy (EDX) are used, it is very unlikely that the bit information stored in these memory cells leak out by imaging attacks.
\end{abstract}

Keywords-hardware security, imaging attacks, SEM, EDX, emerging NVMS, STT-MRAM, RRAM

\section{INTRODUCTION}

Unlike traditional volatile memories (SRAM and DRAM), emerging NVMs [1-2] can retain stored data even when a system's power is off while providing superior scalability. Thus, investigating them as non-volatile components of the memory hierarchy has become a critical field of study. For example, when they are employed as main memory, non-volatility provides the unique benefits of almost zero idle power, and removal of periodic refreshes that would otherwise contribute to a significant portion of power consumption in DRAM-based main memories. However, this can also introduce new design and implementation challenges; and, in particular, for the purposes of this work, we note that critical issues may arise in the hardware security aspect because some sensitive information will remain in the memory and still be able to be probed even when a system's power is off. Information stored in the non-volatile main or cache memory is thus susceptible to various physical attacks, which cannot be protected by conventional software-based countermeasures that only work when a system's power is on. Therefore, the physical attacks from various adversaries may result in unauthorized privilege escalation or significant leakage of sensitive information such as encryption keys and personally identifiable information.

In this study, we chose spin transfer torque magnetic random-access memory (STT-MRAM) [3] and resistive random-access memory (RRAM) [4] as representative emerging NVM devices. Besides the rapidly rising neuromorphic application domain [5-6], both technologies possess the great potential to become a new mainstream memory. The STT-MRAM technology that can endure practically unlimited write/erase programming cycles and therefore fulfill the endurance requirement for main memory ( $>10^{15}$ cycles) has already been developed [7], making it possible to realize visions of replacing DRAM [8], or even certain embedded memories (SRAM cache [9] or eFlash [10]). When it comes to RRAM, owing to its simple device structure and ability to be readily adopted in the bit-cost scalable 3D architecture, RRAM has been the most promising successor to 3D NAND in aggressively scaled technology nodes [11].

Our experiments were conducted on fully-functional, state-of-the-art, nanoscale STT-MRAM and RRAM devices fabricated through our industry collaborators. The security vulnerability of these NVM cells was systematically investigated by programming them to either low-resistance state (LRS) or high-resistance state (HRS) using the semiconductor parameter analyzer (Keithley 4200A) attached to the probe station (Cascade Summit 11000B), ensuring the cell status with low read-out voltages $(0.1 \mathrm{~V})$, and finally examining them in the SEM (Hitachi SU1510) of various operating modes. In regard to the experimental study on physical attacks to memories, traditional volatile (SRAM and DRAM) and non-volatile (ROM and flash) memories have already been explored in terms of fault injection, power analysis, chip modification, microprobing, and imaging attack scenarios [12-13]. This work serves as the security vulnerability study on both emerging NVMs, STT-MRAM and RRAM, under SEM imaging attacks.

\section{SECURITY Vulnerability OF STT-MRAM}

This section examines first the security vulnerability of STT-MRAM by investigating its R-V (resistance-voltage) switching characteristics, programing each cell to either LRS or HRS, and analyzing them in the SEM imaging.

\section{A. STT-MRAM Basics and R-V Characteristics}

In STT-MRAM, a spin-polarized current exerts a spin transfer torque (STT) to change the magnetization direction of a nanomagnet. The resultant resistance difference can be detected as the magnetoresistance (MR) ratio, offering read and write times of down to a few nanoseconds [14]. Recently, Everspin Technologies reported its increasing revenue with Gb STT-MRAM chips that employ the state-of-the-art perpendicular MTJ (pMTJ) technology [15]. Furthermore, using the novel spinorbit torque (SOT) mechanism for magnetization switching may open new possibilities to overcome the shortcomings of contemporary STT-MRAM technology, 
such as the trade-off between switching speed and power consumption [16].

Our STT-MRAM samples feature the cutting-edge p-MTJ technology with a tunneling MR (TMR) ratio of greater than $100 \%$ and a low resistance-area (RA) product of $\sim 10 \Omega \mu \mathrm{m}^{2}$. The p-MTJ stack was deposited on $300 \mathrm{~mm}$ wafers by an Applied Materials' Endura sputtering system. Figure 1 depicts the R-V switching curves measured for two different STT-MRAM cells. Because a recent study predicts that resistance levels of STT-MRAM slightly vary depending on the voltage sweeping details (direction, history and range [5]), we carefully controlled the voltage sweeping experiments in such a way that all memory cells experience the same voltage sweeping event. This ensures that bi-stable bit cells (not in any intermediate states) are programmed in all experiments. It is clearly seen from the figure that the cells switch from LRS to HRS at voltages of around $0.35 \mathrm{~V}$, and transit back to LRS at negatives voltages ranging from -0.2 $\mathrm{V}$ to $-0.5 \mathrm{~V}$. Due to the noted cell-to-cell variation in HRSto-LRS switching voltages, we applied $0.1 \mathrm{~V}$ as a small readout voltage to prevent any accidental switching during read.

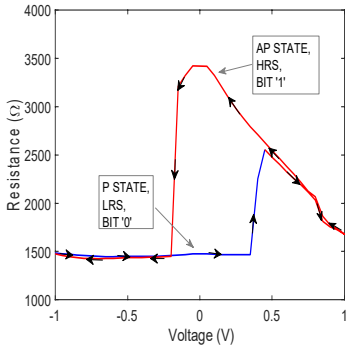

(a)

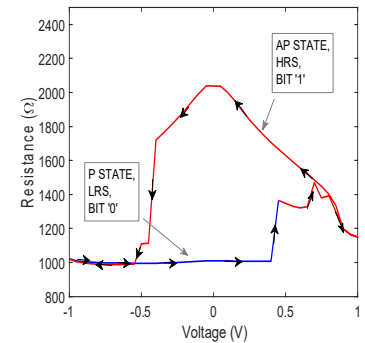

(b)
Fig. 1. R-V switching characteristics of two STT-MRAM cells. The cell shown in (a) has a lower HRS-to-LRS switching voltage than one in (b). Both cells are programmed to either HRS or LRS, to identify any notable difference between HRS and LRS in SEM.

\section{B. SEM Study on STT-MRAM Cells}

SEM is one of the most widely used scientific instruments in microelectronics that scans a focused electron beam over a sample surface to create an image. Modern NVM cells are typically in the order of a few tens to hundreds of nanometers (critical dimensions of our STT-MRAM cells are greater than $50 \mathrm{~nm}$ ), requiring the magnification of at least $5,000 \mathrm{x}$ to obtain the visible image; it is well within the capability of commercial SEM equipment. Obviously, another imaging technique of an optical microscope that is much simpler and cheaper, is not a suitable choice for adversaries to image and decode the modern, nanoscale memory cells. In our experiments, SEM images in the secondary electron (SE) mode were obtained for both STT-MRAM cells in HRS and LRS with acceleration voltages of $20 \mathrm{kV}$ and magnifications ranging from $1,800 x$ to more than $20,000 x$. As shown in Figures 2 and 3 (cells in HRS and LRS, respectively), no significant differences are found in SEM images between STT-MRAM bit cells in HRS and LRS. The active memory storage layer (marked by the yellow square) is not identifiable in the SEM imaging. This is attributed to the fact that the secondary electrons, the primary source of the SEM imaging, are largely generated from the top electrode metallic capping layer, which is far from the active data storage (magnetic free) layer. The STT-MRAM cell typically consists of a large number of magnetic and non-magnetic layers (i.e., the total number of layers in the modern MTJ easily exceeds 10), making the magnetic free layer positioned deep in the middle of the entire device stack and thus less exposed to the direct imaging attack.

Other imaging techniques that have been widely used for observing magnetic microstructures, such as the MOKE (magneto-optical Kerr effect) magnetometry, may also be adopted as a non-destructive imaging method for STTMRAM information readout. The MOKE effect [17] measures the change in the polarization of the reflected light from a magnetic material surface, thus giving access to the magnetization state of the sample. However, in general, since this Kerr effect is also based on the surface magnetism within a relatively small skin depth (less than $20 \mathrm{~nm}$ in most metals for the visible wavelength range used for conventional MOKE), it is still very hard to directly probe the magnetic free (storage) layer that is located deep inside the whole STTMRAM device stack. Ultimately, this implies that the modern STT-RAM technology preserves a high level of security for the non-invasive attack with minimum efforts.

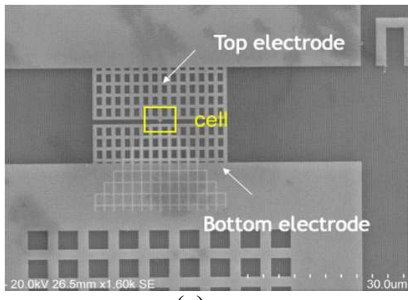

(a)

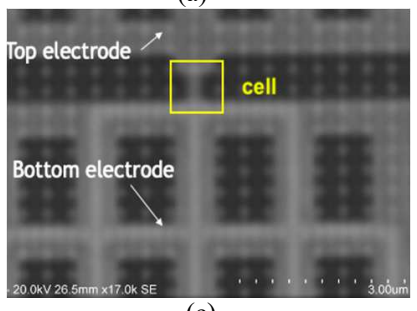

(c)

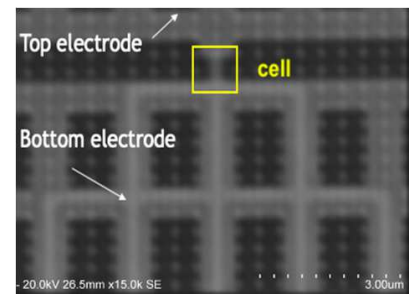

(b)

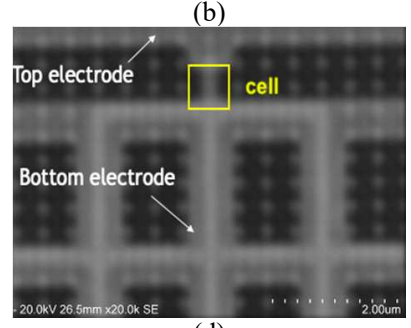

(d)
Fig.2. SEM images of a STT-MRAM cell in HRS at varying magnifications of (a) $1.8 \mathrm{k}$, (b) $15 \mathrm{k}$, (c) $17 \mathrm{k}$, (d) $20 \mathrm{k}$. The cell location is labeled along with top and bottom electrode contacts.

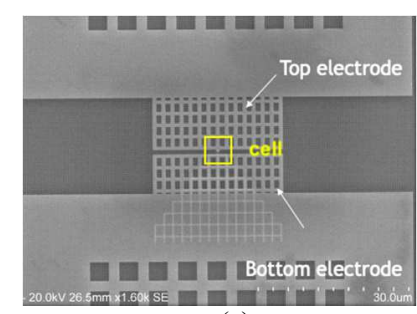

(a)

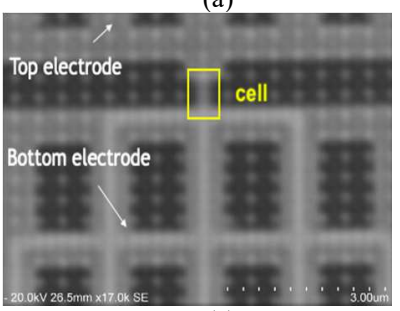

(c)

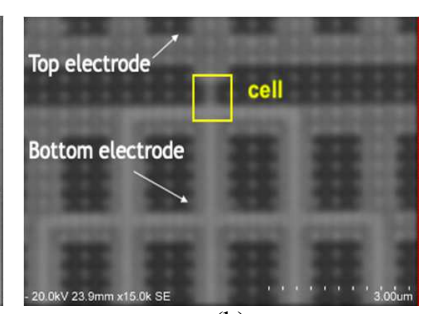

(b)

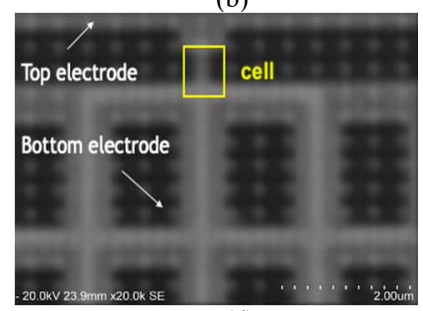

(d)
Fig.3. SEM images of a STT-MRAM cell in LRS at varying magnifications of (a) $1.8 \mathrm{k}$, (b) $15 \mathrm{k}$, (c) $17 \mathrm{k}$, (d) $20 \mathrm{k}$. The cell location is labeled along with top and bottom electrode contacts.

\section{SECURITY VULNERABILITY OF RRAM}

We next apply the similar technique to investigate the security vulnerability of RRAM against the SEM imaging 
attack. As compared with STT-MRAM, RRAM features a relatively simple device structure. Therefore, one may speculate that RRAM is more vulnerable to physical attacks than STT-MRAM. In this study, two different commonly used device geometries of RRAM - namely, capacitor and crossbar, were both examined.

\section{A. RRAM Basics and I-V Characteristics}

RRAM has a simple two-terminal device structure consisting of a transition metal oxide sandwiched between two metal electrodes. RRAM is thus a promising candidate to complement 3D NAND for mass storage applications. The switching mechanism is governed by the formation and rupture of a conductive filament by migrating oxygen ions (metal-oxide RAM) or high-mobility metal electrode ions (conductive-bridge RAM) [4].

In Figure 4, we first examined the hysteretic I-V switching characteristics of two RRAM cells; one is the capacitor-type that exhibits the bipolar behavior (Figure 4a), and another is the crossbar-type (Figure 4b). Because the I-V curves of RRAM are typically characterized by the (possible) existence of a forming curve (the very first SET switching at a relatively high voltage bias) and cycle-to-cycle variability, we tested the RRAM cells with multiple switching cycles until we clearly understand the switching behavior and determine the proper read-out voltages for both LRS and HRS.

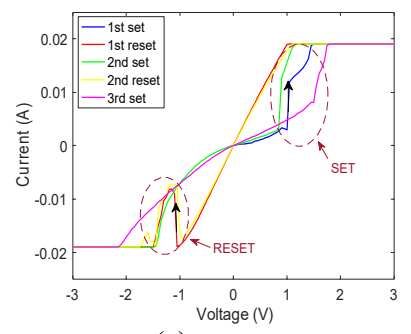

(a)

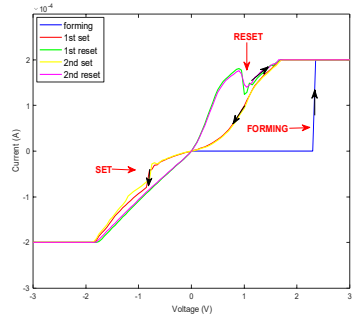

(b)
Fig.4. I-V graphs of RRAM of the capacitor-type (a) and the crossbartype (b). Our capacitor-type RRAM cell (critical dimension: $500 \mathrm{~nm}$ ) exhibits the bipolar switching characteristic along with forming-free behavior. For both devices, multiple switching cycles are confirmed to best determine the cell status and the read-out voltage.

\section{B. SEM Study on RRAM Cells}

After carefully programming both the types of RRAM cells into HRS and LRS, and confirming their bit status, we examined their SEM images using the similar conditions applied to STT-MRAM (SE mode, acceleration voltage of 20 $\mathrm{kV}$, magnifications of up to 12,000x). Figures 5 and 6 show the SEM images of capacitor-type RRAM cells at the active device region for the cell sizes of $500 \mathrm{~nm}$ and $2 \mu \mathrm{m}$, respectively, and Figure 7 depicts the SEM images of the crossbar-type. Despite the relatively simple device structure, RRAM is still found to be very hard to differentiate between LRS and HRS in SE-mode SEM images.

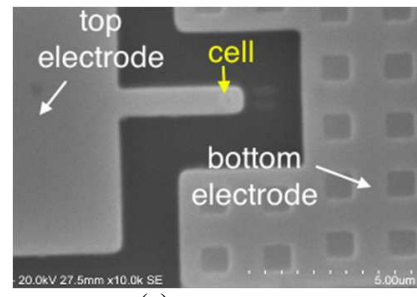

(a)

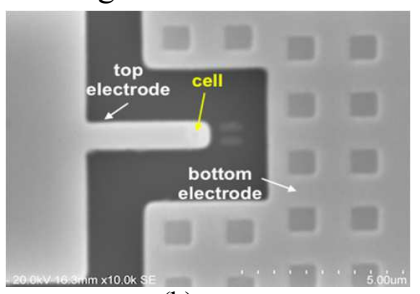

(b)

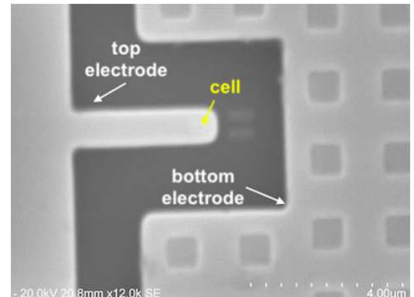

(c)

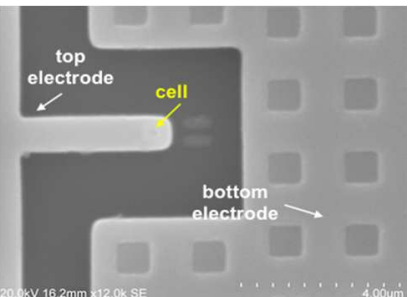

(d)
Fig,5. SEM images of $500 \mathrm{~nm}$ capacitor-type RRAM cells at varying magnifications of (a) 10k (HRS), (b) 10k (LRS), (c) 12k (HRS), (d) $12 \mathrm{k}$ (LRS). No clear differences are observed between cells in HRS and LRS.

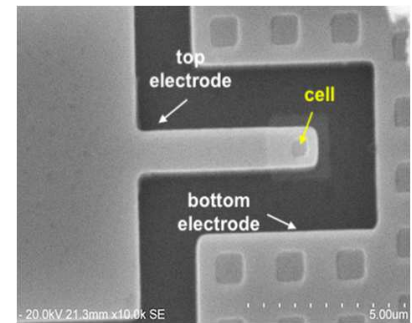

(a)

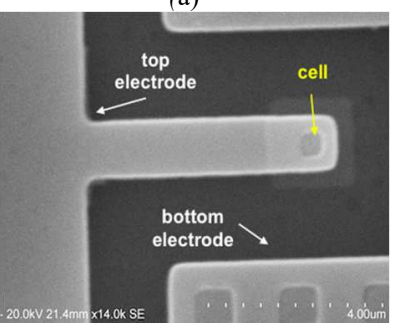

(c)

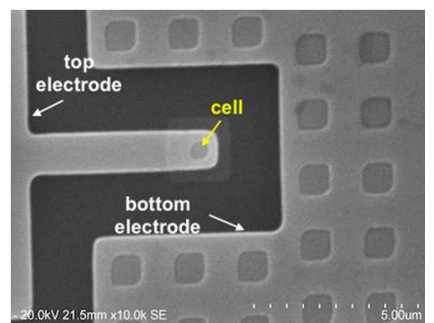

(b)

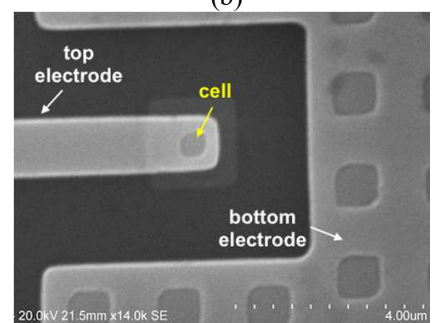

(d)
Fig.6. SEM images of $2 \mu \mathrm{m}$ capacitor-type RRAM cells at varying magnifications of (a)10k (HRS), (b) 10k (LRS), (c) 14k (HRS), (d) 14k (LRS). No clear differences are observed between cells in HRS and LRS.

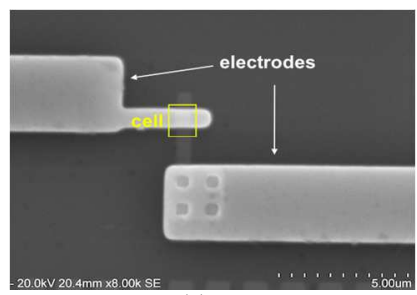

(a)

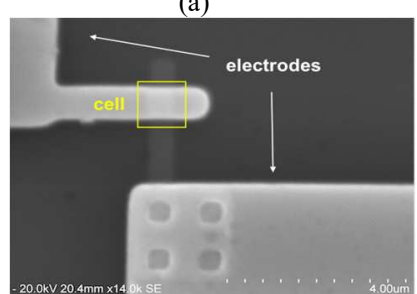

(c)

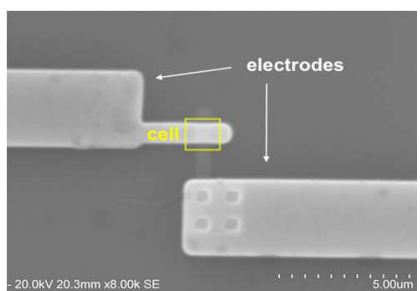

(b)

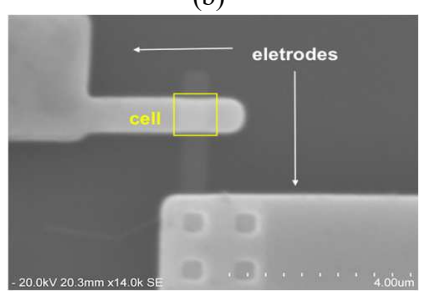

(d)
Fig.7. SEM images of crossbar-type RRAM cells at varying magnifications of (a)8k (HRS), (b) 8k (LRS), (c) 14k (HRS), (d) 14k (LRS). No clear differences are observed between cells in HRS and LRS.

\section{EDX Study on RRAM Cells}

It is noted that the scientific instrument such as SEM is typically equipped with its spectroscopic analysis capability. EDX is a commonly used technique for the elemental or chemical characterization of samples, so adversaries who have access to the SEM equipment may also adopt EDX to further investigate the memory cell. In order to examine the security vulnerability of RRAM under such elemental analysis, we performed EDX on RRAM cells of either LRS or HRS under varying acceleration voltages of SEM. In this task, we focused on the capacitor-type RRAM device that 
shows the bipolar switching behavior, as its switching mechanism has been well understood [4]. According to the accessibility, resources, and time (ART) criteria of Moein and Gebali [18], EDX is still considered as least demanding attack (LDA) for adversaries.

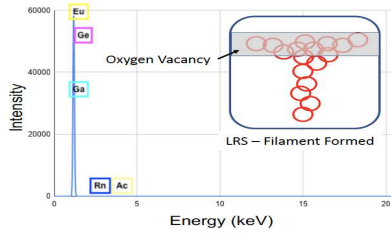

(a)

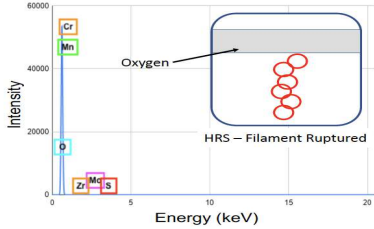

(b)
Fig. 8. EDX results of capacitor-type RRAM cells in LRS (a) and HRS (b). Oxygen is only detected for HRS, where the top surface of the metal oxide has deficit of oxygen vacancies (rupture of a conductive filament).

Figure 8 shows the EDX (with acceleration voltage of 8 $\mathrm{keV}$ ) analysis results on both cells in LRS (a) and HRS (b). We find that oxygen is only detected in the case of HRS. This is explained by the well-known switching mechanism of RRAM. In LRS, the metal oxide layer has a complete conductive filament consisting of oxygen vacancies, and thus, the top surface that is scanned through X-ray reveals no oxygen. On the contrary to this, the RRAM cell in HRS features the filament rupture, leading to recovery of normal metal oxide on the top and consequently, detecting the oxygen element in the EDX analysis. While in perfect agreement with other similar studies [19], this finding clearly indicates that RRAM is vulnerable to such adversaries who possess in-depth understanding of the RRAM's switching mechanism and the EDX. It is important to note that this difference is only detected at relatively low acceleration voltage settings $(6 \mathrm{kV}$ to $10 \mathrm{kV})$. At acceleration voltages of higher than $10 \mathrm{kV}$, oxygen is not found in EDX for both LRS and HRS due to its low atomic number $(Z=8)$. Given that different EDX line scans at different locations still produce the same result, we speculate that the conductive filament of our RRAM devices has the funnel shape (as shown in the Figure 8 inset). Table 1 below summarizes our EDX results.

\begin{tabular}{|l|c|c|c|}
\hline \multirow{2}{*}{ RRAM Cells } & \multicolumn{3}{|c|}{ Acceleration Voltages } \\
\cline { 2 - 4 } & $6 \mathrm{kV}$ & $8 \mathrm{kV}$ & $10 \mathrm{kV}$ \\
\hline $2 \mu \mathrm{m} / \mathrm{LRS}$ & No & No & No \\
\hline $2 \mu \mathrm{m} / \mathrm{HRS}$ & Yes & Yes & Yes \\
\hline $5 \mu \mathrm{m} / \mathrm{LRS}$ & No & No & No \\
\hline $5 \mu \mathrm{m} / \mathrm{HRS}$ & Yes & Yes & Yes \\
\hline
\end{tabular}

Table 1: The EDX analysis results to reveal the oxygen element. No = no oxygen detected, and Yes $=$ oxygen detected.

\section{CONCLUSION}

In this paper, we presented our experimental study on the security vulnerability of emerging NVMs against SEM imaging attacks. Both STT-MRAM and RRAM cells of varying device geometries and sizes were systematically examined in the secondary electron mode SEM. We find that memory cells programmed to LRS and HRS do not exhibit any notable difference in SEM secondary electron mode images with magnifications of up to around 20,000x. This is mainly attributed to the fact that a full-stack NVM device has top electrodes and/or capping layers, requiring more advanced techniques to reveal the bit information stored in the deep, active switching layer. For STT-MRAM, MOKE with deep skin depth may be used to steal the bit information with additional resources and equipment. For RRAM, elemental analysis techniques such as EDX may be used to decode the bit information stored in the metal oxide layer. The methodologies developed in this work can also be applied to investigate other emerging NVMs (e.g., PCM, fRAM) in terms of security vulnerability under imaging attacks.

\section{REFERENCES}

[1] Y. Chen, H. H. Li, I. Bayram, and E. Eken, "Recent technology advances of emerging memories," IEEE Design \& Test, vol. 34, pp. 8-22, June 2017.

[2] S. Yu and P. Chen, "Emerging memory technologies: recent trends and prospects," IEEE Solid-State Circuits Magazine, vol. 8, pp. 43-56, 2016.

[3] X. Fong et al., "Spin-transfer torque memories: devices, circuits, and systems" Proceedings of the IEEE, vol. 104, pp. 1449-1488, July 2016.

[4] D. Ielmini, "Resistive switching memories based on metal oxides: mechanisms, reliability and scaling," Semiconductor Science and Technology, vol. 31, pp. 063002, May 2016.

[5] H. Yan, H. R. Cherian, E. C. Ahn, X. Qian, and L. Duan, "iCELIA: A fullstack framework for STT-MRAM-based deep learning acceleration," IEEE Transactions on Parallel and Distributed Systems, vol. 31, pp. 408, February 2020 .

[6] J. Z. Jiang et al., "Bidirectional analog conductance modulation for RRAMbased neural networks," IEEE Transactions on Electron Devices, vol. 67, pp. 4904, November 2020.

[7] J. J. Kan et al., "Systematic validation of $2 \mathrm{x} \mathrm{nm}$ diameter perpendicular MTJ arrays and MgO barrier for sub-10 nm embedded STT-MRAM with practically unlimited endurance," IEDM (2016).

[8] Y. Jin, M. Shihab, and M. Jung, "Area, power, and latency considerations of STT-MRAM to substitute for main memory," ISCA (2014).

[9] K. Ikegami et al., "Low power and high density STT-MRAM for embedded cache memory using advanced perpendicular MTJ integrations and asymmetric compensation techniques," IEDM (2014).

[10] K. Lee, E. H. Toh, J. T. Wong, and E. K. B. Quek, "STT-MRAM bitcell for embedded flash applications," Google Patents, 2017.

[11] E. C. Ahn, H.-S. P. Wong, and E. Pop, "Carbon nanomaterials for nonvolatile memories," Nature Reviews Materials, vol. 3, pp. 18009, March 2018.

[12] S. Skorobogatov, "How microprobing can attack encrypted memory," Euromicro Conference on Digital System Design (2017).

[13] F. Courbon, S. Skorobogatov, and C. Woods, "Reverse engineering flash EEPROM memories using scanning electron microscopy," University of Cambridge, Springer International Publishing, 2017, p. 57-72.

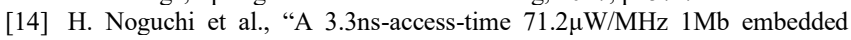
STT-MRAM using physically eliminated read-disturb scheme and normally-off memory architecture," ISSCC (2015).

[15] MRAM-Info, "Everspin reported its preliminary Q4 2020 and full-year 2020 financial results," website, 2021.

[16] K. Narayanapillai et al., "Spin-transfer versus spin-orbit torque MRAM," INEC (2016).

[17] Z. Q. Qiu and S. D. Bader, "Surface magneto-optic Kerr effect," Review of Scientific Instruments, vol. 71, pp.1243-1255, March 2000

[18] S. Moein and F. Gebali, "Quantifying covert hardware attacks: using ART schema," Computer Science and Its Applications pp. 511, Springer (2015).

[19] J.F. Konopka, "Quantitative Analysis of Oxygen in Metal Oxides with SEM/EDS by Direct Measurement of all X-ray Peaks", American Geophysical Union, Dall Meeting 2012, December 2012. 